\title{
A ATENÇÃO DA ENFERMEIRA À SAÚDE CARDIOVASCULAR DE MULHERES HIPERTENSAS
}

\author{
The nurse care of cardiovascular health in hypertense women \\ La atención de la enfermera en la salud cardiovascular de mujeres \\ con hipertensión
}

\author{
Luana Santos de Assis \\ Joséte Luzia Leite ${ }^{3}$
}

Marluci Andrade Conceição Stipp 2

Natália Machado da Cunha ${ }^{4}$

\section{RESUMO}

Este estudo é parte integrante da análise dos resultados do Projeto Integrado de Pesquisa intitulado Prevalência das Doenças Cardiovasculares na Clientela atendida num Hospital Escola do Município do Rio de Janeiro. Estudo descritivo, exploratório, quantitativo. Objetivos: estimar a frequência de hipertensão arterial na clientela feminina atendida no ambulatório de um hospital escola do município do Rio de Janeiro, traçar o perfil de risco para as doenças cardiovasculares dessa clientela e elaborar um plano de intervenções baseado em seu perfil. Foi aplicado um questionário a 102 clientes. Encontramos 64,71\% hipertensas; destas, 89,39\% faziam uso de alguma medicação anti-hipertensiva, 68,18\% referiram não praticarem exercícios físicos regularmente; 54,55\% consideram-se estressadas; e 72,73\% apresentavam história familiar de doença cardiovascular. A atenção à clientela hipertensa objetiva, além do controle dos padrões de estilo de vida, o controle da pressão arterial, a investigação sobre os fatores de risco e a orientação sobre a medicação usada e seus efeitos.

Palavras-chave: Enfermagem. Saúde da Mulher. Doenças Cardiovasculares.

\begin{abstract}
This study is part of the data analysis of the Integrated Project named Cardiovascular Disease Prevalence in the Clientele examined at Rio de Janeiro's Municipal School Hospital. The referred study is descriptive, exploratory and quantitative. Objectives: To estimate the frequency of arterial hypertension in a female clientele assisted in the ambulatory of the School Hospital; to elaborate the risk profile for cardiovascular disease of the referred clientele, and to develop an intervention plan based on the clientele's profile. A questionnaire was applied in 102 patients. It was found that $64,71 \%$ were hypertensive; $89,39 \%$ of whom used some kind of medication against Hypertension. From the patients above, $68,18 \%$ mentioned absence of regular exercise practices, $54,55 \%$ considered themselves stressed out and $72,73 \%$ had a family history of cardiovascular disease. The care to the hypertense clientele aims at the control of life standard patterns, blood pressure monitoring, risk factors investigations, and counseling of medication and its effects.
\end{abstract}

Keywords: Nursing. Women's health. Cardiovascular diseases.

\section{Resumen}

Este estudio es parte integrante del análisis de los resultados del Proyecto Integrado denominado Prevalencia de las Enfermedades Cardiovasculares en la Clientela examinada en el Hospital Escuela del Municipio del Rio de Janeiro. Estudio descriptivo, exploratorio y cuantitativo. Objetivos: Estimar la frecuencia de la hipertensión arterial en la clientela femenina asistida en el ambulatorio de un Hospital Escuela del Municipio del Rio de Janeiro, trazar el perfil de riesgo para las enfermedades cardiovasculares de la clientela referida, y desarrollar un plan de intervención basado en el perfil de la clientela. Un cuestionario fue aplicado en 102 clientes. Encontramos $64,71 \%$ hipertensas; de estas $89,39 \%$ utilizó alguna medicación contra la hipertensión, $68,18 \%$ mencionaron no practicar ejercicios físicos regularmente, $54,55 \%$ se consideraba tensionadas, $72.73 \%$ tenían antecedentes familiares de la enfermedad cardiovascular. La atención a la clientela hipertensa tiene como objetivo más allá del control de los patrones del estilo de vida, el control de la presión arterial, investigación sobre los factores de riesgo y orientación sobre la medicación usada y sus efectos.

Palabras clave: Enfermería. Salud de la mujer. Enfermidades cardiovasculares

${ }^{1}$ Acadêmica do $6{ }^{0}$ período de Enfermagem EEAN/UFRJ. Bolsista IC da FAPERJ. Brasil. E-mail: luanas-assis@hotmail.com, ${ }^{2}$ Prof. ${ }^{a}$ Adjunta do Departamento de Metodologia da Enfermagem da EEAN/UFRJ. Membro da Diretoria do Núcleo de Pesquisa Gestão em Saúde e Exercício

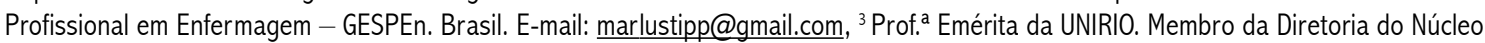
de Pesquisa Gestão em Saúde e Exercício Profissional em Enfemagem da EEAN/UFRJ - GESPEn. Brasil. E-mail: joluzia@gmail.com, ${ }^{4}$ Acadêmica do $7^{\circ}$ período de Enfermagem da EEAN/UFRJ; Bolsista PIBIC/CNPq. Brasil. E-mail: nataliacunha@hotmail.com 


\section{INTRODUÇÃO}

Nos últimos tempos, o avanço tecnológico e industrial trouxe várias consequências para população, na área da saúde, resultando em uma mudança no seu perfil de morbimortalidade, com grande predomínio das doenças e mortes devidas às doenças crônicas não transmissíveis (DCNT), entre elas as doenças cardiovasculares'.

As doenças cardiovasculares têm sido a causa mais comum de mortalidade no Brasil e no mundo, conforme Datasus ${ }^{2}$. Segundo esses dados, a mortalidade por doenças do aparelho circulatório no Brasil é de 74.216 óbitos, sendo 36.876 do sexo feminino. Houve, neste mesmo ano, 10.991 óbitos em mulheres por doenças isquêmicas do coração, 12.165 óbitos por doenças cerebrovasculares e 5.902 óbitos por diabetes mellitus.

As mulheres são a maioria da população brasileira, 50,77\%, e as principais usuárias do Sistema Único de Saúde (SUS). Elas frequentam os serviços de saúde como usuárias, mas também como acompanhantes de crianças e outros familiares, pessoas idosas, com deficiência, vizinhos, amigos ${ }^{3}$. São também cuidadoras, não só das crianças ou outros membros da família, mas também de pessoas da vizinhança e da comunidade 3 .

A saúde cardiovascular envolve muitos aspectos, como o lazer, a alimentação, as condições de trabalho e moradia. A saúde cardiovascular da mulher é uma questão merecedora de atenção, pois ela enfrenta uma sobrecarga de responsabilidades com o trabalho e as atividades domésticas, e isso pode ter um impacto na sua saúde. Alguns questionamentos são feitos em torno da temática Saúde da Mulher, como por exemplo: Por que a Mulher adoece mais? As atividades profissionais, juntamente com as atividades do lar e o cuidar dos filhos, configuram uma tripla jornada de trabalho; este aspecto favorece esse adoecimento?

No âmbito das doenças cardiovasculares, destaca-se a hipertensão arterial, que é a mais prevalente das doenças cardiovasculares e também o principal fator de risco para as complicações mais comuns, como acidente vascular cerebral e infarto agudo do miocárido ${ }^{1}$. No Brasil são cerca de 17 milhões de portadores de hipertensão arterial; esse número é crescente, a carga de doenças representada pela morbimortalidade devida à doença é muito alta, e por tudo isso a hipertensão arterial é um problema grave de saúde pública no Brasil e no mundo'.

Partindo dessas questões, o foco deste estudo foi o perfil de risco de mulheres hipertensas. Os objetivos foram estimar a frequência de hipertensão arterial na clientela feminina atendida no ambulatório de um hospital escola do município do Rio de Janeiro, traçar o perfil de risco para as doenças cardiovasculares dessa clientela e elaborar um plano de intervenções baseado nesse perfil da clientela atendida.

$\mathrm{Na}$ atualidade, uma visão simplista da doença cardiovascular na mulher não mais se justifica, a doença coronária tornou-se a principal causa de morte no sexo feminino do mundo ocidental, maior que o câncer de útero, de mama ou mortes no parto ${ }^{4}$.
A mudança do padrão de vida das mulheres talvez explique, em parte, a ocorrência de dados tão alarmantes: ao lado das responsabilidades tradicionais com a casa, filhos, marido e parentes idosos, as mulheres adquiriram as responsabilidades que antes se destinavam ao homem; trabalho fora do lar e necessidades financeiras decorrentes de abandono, divórcio e viuvez são exemplos disso. Ao mesmo tempo, as mulheres adquiriram "hábitos de homem": dietas irregulares e sem restrição de gorduras e carboidratos, fumo, álcool, falta de atividade física regular e de repouso adequado. ${ }^{4}$

Assim, a alta prevalência na população brasileira, as consequências e seu elevado custo para o sistema de saúde justificam a elaboração deste estudo.

\section{MÉTODOS E TÉCNICAS}

Este estudo é parte integrante da análise dos resultados oriundos do Projeto Integrado de Pesquisa intitulado "Prevalência das Doenças Cardiovasculares na Clientela atendida num Hospital Escola do Município do Rio de Janeiro". Este projeto está em andamento e está sendo desenvolvido no hospital escola São Francisco de Assis da Universidade Federal do Rio de Janeiro. É subprojeto de um projeto "guarda-chuva" desenvolvido pelo grupo de pesquisa intitulado "Enfermagem Cardiovascular: Aspectos gerenciais e de educação em saúde", registrado no diretório de grupo de pesquisa do Conselho Nacional de Desenvolvimento Científico e Tecnológico (CNPq).

A população constituiu de clientes atendidos ambulatorialmente atendidos na referida instituição. Os critérios para elegibilidade dos indivíduos do estudo foram: clientes do sexo feminino, acima de 18 anos, em condições clínicas de se comunicar, que buscavam atendimento ambulatorial nas diversas especialidades clínicas do hospital e que aceitaram participar mediante apresentação e assinatura do Termo de Consentimento Livre e Esclarecido.

Foi assegurado às participantes sigilo quanto às informações prestadas, de acordo com a Resolução n ${ }^{0}$ 196/06 do Conselho Nacional de Saúde. A pesquisa foi aprovada pelo Comitê de Ética e Pesquisa da Escola de Enfermagem Anna Nery (EEAN) e Hospital Escola São Francisco de Assis (HESFA) da Universidade Federal do Rio de Janeiro (UFRJ), protocolo $n^{\circ}$ 044/77.

0 instrumento para coleta de dados foi um questionário aplicado oralmente com perguntas sobre o perfil de risco para as doenças cardiovasculares: idade, sexo, história familiar, obesidade, hipertensão arterial, diabetes mellitus, dislipidemia, tabagismo, etilismo, sedentarismo, estresse, menopausa, reposição hormonal.

Os dados foram coletados pelos membros da equipe do projeto no ambulatório, no período da manhã e da tarde, de junho de 2007 a maio de 2008. Os clientes foram escolhidos de forma aleatória enquanto aguardavam na sala de espera a consulta dos profissionais de saúde. Os resultados foram 
tabulados e organizados utilizando-se o método de estatística descritiva, de frequência simples e percentual.

Antes da aplicação do instrumento, foi realizado um treinamento com a pesquisadora responsável e os alunos de Iniciação Científica inseridos no projeto, para que os mesmos aplicassem o questionário de forma uniformizada, garantindo a correta coleta das informações. Foi realizado um pré-teste para assegurar validade e precisão do instrumento.

\section{APRESENTAÇÃO DOS RESULTADOS}

Participaram deste estudo até o momento 102 mulheres, sendo 66 hipertensas, o que corresponde a $64,71 \%$. Dessas, 89,39\% fazem uso de algum medicamento anti-hipertensivo, com o enalapril, captopril, hidroclorotiazida e atenolol entre os mais citados. No que diz respeito ao tempo de acompanhamento ambulatorial dos entrevistados, foi visto que $36,36 \%$ têm o diagnóstico de hipertensão arterial num período que varia de 6 a 10 anos, seguidos de $30,30 \%$ no período de 1 a 5 anos; 15,15\%, no período de 11 a 20 anos; e 9,09\%, no período de mais de 20 anos; $6,06 \%$ realizam acompanhamento com menos de um ano, e apenas 3,03\% não souberam informar.

Quanto às mulheres hipertensas, a maioria se concentrou na faixa etária entre 41 e 60 anos, perfazendo um total de $54,55 \% .56,06 \%$ são naturais da cidade do Rio de Janeiro, $57,58 \%$ apresentam vida conjugal e $63,64 \%$ se autodeclararam da cor negra ou parda.

Em relação ao trabalho, 39,39\% trabalham e são remuneradas; dentre as ocupações mais citadas, observamos auxiliar de serviços gerais, doméstica, costureira e dona de casa. 60,61\% e 63,64\% apresentam renda individual e renda familiar de 1 e 3 salários mínimos, respectivamente. E quanto à escolaridade, 63,64\% têm ensino fundamental incompleto.

Foi visto que $48,48 \%$ são as principais responsáveis pela renda da família. 69,70\% moram em casa própria, 93,94\% têm água encanada, 95,45\% têm rede de esgoto, e $100 \%$ das entrevistadas referiram possuir em suas residências coleta de lixo e luz elétrica.

Algumas características importantes no estudo das doenças crônicas, como variáveis de renda, ocupação, escolaridade, classe social e outras, vêm sendo utilizadas para identificação de determinados grupos específicos, Isso auxilia na identificação dos aspectos ligados à melhoria das condições de saúde e de vida para quem convive com problemas cardiovasculares, influenciando no estilo de vida das pessoas 5 .

Com relação ao acesso a uma Unidade Básica de Saúde, $77,27 \%$ relataram ter fácil acesso ao atendimento de saúde. Sobre a alimentação e ingesta hídrica, 39,39\% realizam quatro refeições diárias e 30,30\% bebem mais de seis copos de 200 $\mathrm{ml}$ de água por dia, o que corresponde a mais de $1.200 \mathrm{ml}$.

0 estilo de vida pode influenciar positivamente na situação de bem-estar e saúde de pessoas portadoras de hipertensão arterial. Sobre o perfil de risco cardiovascular, identificamos alguns fatores de risco mais prevalentes; $68,18 \%$ referiram não praticar exercícios físicos regularmente, 54,55\% consideraram-se estressadas; $72,73 \%$ relataram história familiar de doença cardiovascular.

Em relação à gordura centrípeta, 59,09\% apresentaram a circunferência abdominal acima do padrão normal; de acordo com a I Diretriz Brasileira de Diagnóstico e Tratamento da Síndrome Metabólica ${ }^{6}$, a obesidade abdominal para as mulheres seria a circunferência abdominal com valor maior ou igual a 88 cm; 39,39\% apresentam o Índice de Massa Corporal (IMC) com valor entre 25 e 29,9, o que está relacionado ao aumento de peso; 59,09\% apresentam elevação no colesterol; e 62,12\% estão na menopausa.

Apenas 19,70\%, relataram ingerir bebida alcoólica. Com relação à hipertensão arterial, a restrição da ingestão de álcool é uma medida eficaz na redução da pressão arterial ${ }^{7}$.

É importante destacar que, apesar da alta prevalência de diabetes mellitus na população brasileira, apenas 18,18\% dessas mulheres apresentaram este tipo de morbidade.

\section{DISCUSSÃO DOS DADOS}

A hipertensão arterial é definida como a pressão arterial sistólica maior ou igual a $140 \mathrm{mmHg}$ e a pressão arterial diastólica maior ou igual a $90 \mathrm{mmHg}$, em indivíduos que não estão fazendo uso de medicação anti-hipertensiva. Assim, 0 limite escolhido para definir a hipertensão ar terial é igual a 140/90 mmHg quando encontrado em pelo menos duas aferições realizadas em momentos diferentes ${ }^{8}$. Porém, no acompanhamento clínico, são indispensáveis a realização do exame físico e uma avaliação cardiovascular global.

Como observado, a frequência de hipertensão arterial na população feminina da referida instituição foi bem elevada. № Brasil, a estimativa de prevalência da hipertensão arterial varia de 22,3\% a 43,9\% de acordo com a área estudada ${ }^{8}$.

Em nosso País, há uma grande homogeneidade em relação a etnias, isso requer atenção, visto que não se aborda adequadamente os problemas pertinentes a cada grupo étnicocultural de maneira mais aprofundada. Neste estudo, foi encontrado um elevado número de mulheres da cor negra ou parda, e isso pode ter uma forte relação com a alta frequência de hipertensão arterial, já que a etnia negra é um for te fator predisponente à hipertensão arterial, como também a um maior risco de ataque cardíaco e morte súbita, quando comparada à etnia branca9.

Percebemos que a grande maioria é trabalhadora, tem baixa renda e baixo nível de escolaridade. De acordo com os dados do Instituto Brasileiro de Geografia e Estatística (IBGE) de 2000" no que se refere às diferenças nos níveis educacionais alcançados entre homens e mulheres, percebeu-se que, nos níveis mais baixos de escolaridade, as mulheres aparecem em maiores proporções, mas a tendência é de que, com o tempo, esse quadro se inverta. As mulheres estão tendo mais acesso à escola do que antigamente e, por sua vez, estão nela permanecendo por mais tempo. 
O trabalho doméstico foi uma das ocupações que mais foram citadas. É importante ressaltar esta questão, visto que podemos considerá-lo como um trabalho não remunerado, que "gasta" um tempo a mais além das outras atividades desenvolvidas pelas mulheres.

As mulheres, muito mais do que os homens, dedicam parte significativa do seu tempo aos afazeres domésticos; são elas que mais trabalham atualmente na atividade produtiva, no domicílio e na atividade reprodutiva, enfrentando enorme sobrecarga de trabalho e dificuldades de conciliação entre as responsabilidades familiares e as profissionais ${ }^{11}$.

Embora, no Brasil, ainda haja problemas referentes às moradias inadequadas e falta de saneamento básico, principalmente em regiões da periferia e áreas rurais, a grande maioria da população deste estudo não é acometida por esta problemática. Podemos dizer que as questões de moradia e saneamento básico são importantes indicadores no que se refere à qualidade de vida dos grupos humanos; elas demonstram 0 reconhecimento ao direito básico do cidadão reafirmado pelo Estado, e a igualdade no direito à saúde ${ }^{12}$.

No que se refere à esfera familiar, a sociedade brasileira passou por algumas transformações; como podemos observar no estudo, a maior parte das mulheres são as principais responsáveis pela renda familiar. Segundo os dados do IBGE de $2000^{10}, 24,9 \%$ dos domicílios tinham mulheres como responsáveis. Isso acarreta maior responsabilidade do sexo feminino pelo orçamento da casa, contribuindo para o aumento do nível de estresse, ansiedade, refletido em outras esferas como a social e da qualidade de vida, e o surgimento de um estilo de vida prejudicial, como má alimentação, sedentarismo, aumento de peso corporal, entre outros.

No estudo de Gadelha ${ }^{13}$, foi observado que a ocorrência de hipertensão arterial esteve fortemente ligada ao excesso de peso. Demonstrou-se ainda que, em uma população urbana de baixa renda, a massa corporal é um importante determinante da elevação da pressão arterial, visto que os dois fatores estão fortemente associados.

No que diz respeito à atividade física, esta tem um efeito hipotensor na pressão arterial, além de auxiliar no controle de outros fatores de risco como excesso de peso e dislipidemias, reduzindo assim o risco cardiovascular global. Níveis elevados de colesterol, juntamente com hipertensão arterial, representam mais que $50 \%$ do risco atribuível para doença coronária ${ }^{14}$.

Um dos maiores problemas da sociedade atualmente, é estar sob constante ação de eventos estressantes, seja no âmbito familiar, no trabalho ou nas ruas. Isso pode ser constatado neste estudo, no qual a maioria das mulheres se considerou estressada. 0 sistema cardiovascular participa ativamente das adaptações ao estresse, ocasionando respostas que culminam, principalmente, em aumento da frequência cardíaca, da contratilidade, do débito cardíaco e da pressão arterial ${ }^{15}$.
Outra questão merecedora de atenção foi o fácil acesso dos clientes entrevistados a uma Unidade Básica de Saúde. A Unidade Ambulatorial do HESFA não atende apenas a uma área programática, mas sim a uma demanda espontânea. São clientes oriundos de diversos locais do município do Rio de Janeiro, de outras instituições e dos programas assistenciais desenvolvidos no próprio HESFA.

0 HESFA dispõe de tratamentos alternativos como ambulatórios de homeopatia e acupuntura, além de contar com assistência primária e secundária nas diversas especialidades clínicas, como consultas de enfermagem nas áreas de ginecologia primária (preventivo de colo uterino e mama), doenças sexualmente transmissível e pré-natal, de acordo com os protocolos do Ministério da Saúde.

A atenção ambulatorial para uma clientela hipertensa objetiva não apenas o controle dos padrões de estilo de vida, biológicos e socioculturais. É importante também controlar a pressão arterial e a frequência cardíaca; investigar sobre os fatores de risco, assim como os hábitos de vida; avaliar os sinais e sintomas da doença crônica; e orientar os familiares e a clientela sobre a medicação usada e seus efeitos. 0 trabalho da enfermeira no ambulatório abrange também outras atividades ligadas à gerência do serviço de enfermagem e ao controle das atividades de orientação e supervisão de técnicos e/ou auxiliares de enfermagem sob sua liderança ${ }^{14}$.

0 plano de intervenção de enfermagem para essa clientela seria uma estratégia que objetivasse diminuir as complicações ocasionadas pela hipertensão arterial. A abordagem multiprofissional da clientela com hipertensão arterial é muito importante, pois trata-se de uma doença cujo tratamento tem vários objetivos a serem alcançados nos três diferentes níveis assistenciais (primário, secundário e terciário), permitindo assim uma atuação mais ampla no tratamento.

Tendo como base as V Diretrizes Brasileiras de Hipertensão Arterial $^{14}$, as ações do Plano Assistencial de Enfermagem seriam: consulta de enfermagem, incluindo medida da pressão arterial, da altura e do peso, da circunferência da cintura e do quadril e cálculo do índice de massa corporal; investigação sobre fatores de risco e hábitos de vida; orientação sobre a doença e o uso regular de medicamentos prescritos pelo médico; e orientaç̃̃es sobre hábitos de vida pessoais e familiares, incluindo controle do peso, melhora no padrão alimentar, redução do consumo de sal, moderação no consumo de álcool e realização de exercício físico.

Um plano de intervenção também abarca as atividades de educação em saúde, que podem ser realizadas em salas de espera ou em pequenos grupos, como atividades de grupoterapia. As orientações de enfermagem também podem ser feitas por meio das visitas domiciliares, como preconizado no Programa de Saúde da Família.

Todas essas aç̃̃es têm como objetivo melhorar a adesão ao tratamento, visto que, por se tratar de uma doença crônica e sem sintomas aparentes, muitas vezes ocorre abandono do 
tratamento. As variáveis relacionadas à pessoa hipertensa, como idade, sexo, escolaridade, nível socioeconômico, ocupação, estado civil, hábitos de vida, aspectos culturais e crenças de saúde, são fatores que interferem na adesão ao tratamento ${ }^{16}$; um paciente com baixa condição socioeconômica terá uma limitação na aquisição de medicamentos.

Entendemos que um plano de intervenções para uma clientela com o perfil encontrado é aquele que compõe a etapa do planejamento ou prescrição de enfermagem da metodologia da assistência.

Esta etapa é subsidiada pelas etapas anteriores e embasa a continuidade do processo de enfermagem. Para que seja efetiva e eficaz, deve ter por base o histórico de enfermagem atualizado, além de considerar sempre o dinamismo do processo saúdedoença que o indivíduo a ser cuidado está vivenciando ${ }^{17}$.

0 planejamento deve ter o foco nos problemas da clientela, evidenciando: os objetivos do tratamento, ingesta líquida e hábitos alimentares adequados, respeitando-se o descrito nas diretrizes já mencionadas ${ }^{6,14}$; regularidade nas atividades físicas, sobretudo nas atividades aeróbicas; orientações a respeito da ação e efeitos da medicação prescrita; e atividades de educação em saúde para reforço das orientações da consulta de enfermagem, sobretudo da convivência com fatores de risco cardiovascular.

Nas atividades de educação em saúde, é importante uma metodologia da assistência de enfermagem para grupos, com planejamento para cada encontro, elaborada de forma particularizada, considerando-se o que se tem coletado de informações e as situações vivenciadas em encontros anteriores ${ }^{17}$.

Para esta clientela, deve-se ressaltar o cuidado de saúde no domicílio, no caso a visita domiciliar, atentando para as suas condições socioeconômicas e culturais, a convivência com fatores de riscos, as descobertas de pontos positivos e possíveis dificuldades nas resoluções de problemas de saúde.

É necessário que o cuidado domiciliar seja discutido e planejado por todos os integrantes da equipe de saúde, que 0 estabeleça da maneira que alcance o levantamento de problemas, verificando potencialidades e fragilidades nas suas resoluções, propondo soluções às partes envolvidas neste processo e um plano de ação que fique sujeito a reavaliações periódicas ${ }^{17}$.

\section{CONSIDERAÇÕES FINAIS}

Diante dos resultados obtidos, percebemos que a hipertensão arterial acomete grande parte da clientela atendida e que, concomitantemente, são encontrados outros fatores de risco como estresse, sobrepeso, dislipidemia e sedentarismo. A maior parte faz uso de medicação anti-hipertensiva e um acompanhamento ambulatorial da doença e seus fatores de risco. Um fator importante também relacionado à questão de gênero é que trabalhamos com uma clientela feminina hipertensa que procura uma assistência de saúde. Como já sabemos, as mulheres têm um histórico de frequentar mais os serviços de saúde; além disso, recebem atendimento nas diversas especialidades, como intervenções educativas de prevenção de agravos de doenças crônicas degenerativas, entre outras.

Esforços devem ser feitos para a redução da morbimortalidade oriunda das doenças cardiovasculares, principalmente investir na prevenção primária e promoção da saúde. Por meio da consulta de enfermagem, identificamos os fatores de risco cardiovasculares e as complicações da hipertensão arterial, além de realizarmos a educação em saúde, que constitui um dos principais elementos para melhorar as condições de vida de pessoas portadoras de doenças cardiovasculares.

A monitoração dos fatores de risco pela enfermeira é de suma importância, pois auxilia na identificação dos aspectos ligados à melhoria das condições de saúde e de vida para quem convive com problemas cardiovasculares ${ }^{5}$.

É importante que haja um processo de educação e reeducação da clientela que convive com os fatores de risco cardiovasculares, com a finalidade de preservar a saúde e bem-estar e a qualidade de vida das pessoas.

\section{Referências}

1- Ministério da Saúde (BR). Caderno de Atenção Básica-Hipertensão Arterial Sistêmica. $1^{\mathrm{a}}$ ed. Brasília (DF); 2006.

2- DATASUS. Sistema de informações sobre mortalidade.[ acesso 2008] Disponível em http://tabnet.datasus.gov.br/cgi/idb2006/ matriz.htm\#mort.

3- Ministério da Saúde (BR). Política Nacional de Atenção Integral à Saúde da Mulher: princípios e diretrizes. $1^{\text {a} e d . ~ B r a s i ́ l i a ~(D F) ; ~} 2007$.

4- Luz PL, Solimene MC. Peculiaridades da doença arterial coronária na mulher. Rev Ass Med Bras 1999. 45(1): 45-54.

5- Stipp MAC, Cunha NM. Risco cardiovascular numa clientela ambulatorial: um estudo quantitativo. Online Braz J Nurs [on-line]. 2008 jan. Disponível em: http://www.uff.br/objnursing/index.php/ nursing/article/viewArticle/j.1676-4285.2008.1213/289

6- Sociedade Brasileira de Cardiologia. Sociedade Brasileira de Endocrinologia e Metabologia. Sociedade Brasileira de Diabetes. $1^{\text {a }}$ Diretriz Brasileira de Diagnóstico e Tratamento da Síndrome Metabólica. Arq Bras Cardiol 2005 (supl 1): 1-28.

7 - Stipp MAC, Leite JL, Cunha NM, Assis LS, Andrade MP, Simões RD. 0 consumo do álcool e as doenças cardiovasculares: uma análise sob o olhar da enfermagem. Esc Anna Nery Rev Enferm 2007dez; 11(4): 581-85.

8 - Sociedade Brasileira de Cardiologia. Sociedade Brasileira de Hipertensão. Sociedade Brasileira de Nefrologia. $4^{\mathrm{a}}$ Diretriz Brasileira de Hipertensão Arterial. Arq Bras Cardiol 2006.(supl 5): 1-48.

9- Cruz ICF, Lima R. Etnia negra: um estudo sobre a Hipertensão Arterial Essencial

-HAE e os fatores de risco cardiovasculares. Rev Enferm UERJ 1999; 35-44. 
10- Instituto Brasileiro de Geografia e Estatística-IBGE [on-line]. [acesso 2008]. Disponível em:

http://www.ibge.gov.br/home/estatistica/populacao/perfildamulher/ default.shtm

11- Bruschini C. Trabalho doméstico: inatividade econômica ou trabalho não-remunerado? Rev Bras Estud Popul 2006;. 331-53.

12-Stipp MAC. A saúde da mulher e os fatores de risco coronarianos: práticas de bem-estar e condições de qualidade de vida - indicativos para assistência de enfermagem ambulatorial. [tese de doutorado] Rio de Janeiro (RJ); Escola de Enfermagem Anna Nery. UFRJ; 2000.

13- Gadelha FV, Feijão AMM, Bezerra AA, Oliveira AM, Silva MSS, Lima JWO. Prevalência de excesso de peso e hipertensão arterial, em população urbana de baixa renda. Arq Bras Cardiol 2005; 84(1): 2933.

14 - Sociedade Brasileira de Cardiologia. $4^{\text {a }}$ Diretriz Brasileira sobre Dislipidemias e

Prevenção da Aterosclerose. Arq Bras Cardiol 2007 (supl 1): 1- 18. 15 - Loures DL, Sant'Anna I, Baldotto CSR, Sousa EB, Nóbrega ACL. Estresse mental e sistema cardiovascular. Arq Bras Cardiol 2002; 78(5): 525-30.

16 - Nobre F, Pierin AMG, Mion D. Adesão ao tratamento o grande desafio da hipertensão. São Paulo (SP): Lemos Ed;, 2001.

17- Westphalen MEA, Carraro TE. Metodologias para a assistência de enfermagem: Teorizações, modelos e subsídios para a prática. Goiânia(GO): AB; 2001. 